\title{
Coarsened Exact Matching of Trabectome Surgery Combined with Baerveldt to Baerveldt: Same Session Trabectome Negates Tube Fenestration
}

Hamed Esfandiari, ${ }^{1,2}$ Kiana Hassanpour, ${ }^{2}$ Peter Knowlton, ${ }^{1}$ Tarek Shazly, ${ }^{1}$ Mehdi Yaseri, ${ }^{3}$ Nils A. Loewen ${ }^{1 *}$

${ }^{1}$ Department of Ophthalmology, School of Medicine, University of Pittsburgh, Pittsburgh, Pennsylvania, United States

${ }^{2}$ Ophthalmic Research Center, Shahid Beheshti University of Medical Sciences, Tehran, Iran

${ }^{3}$ Department of Epidemiology and Biostatistics, School of Public Health, Tehran University of Medical Sciences, Tehran, Iran

*Correspondence to Nils Loewen, MD, PhD

203 Lothrop St, Suite 819, Pittsburgh, PA 15213

Department of Ophthalmology, School of Medicine, University of Pittsburgh, Pittsburgh, Pennsylvania, United States.

Email: loewen.nils@gmail.com 


\section{Abstract}

Purpose: To evaluate the efficacy and survival rates of trabectome-mediated ab interno trabeculectomy combined with non-fenestrated Baerveldt glaucoma implants (BT) in comparison to Baerveldt glaucoma implant alone (B).

Method: A total of 175 eyes undergoing primary glaucoma surgery (60 eyes BT and $115 \mathrm{~B}$ ) were enrolled in this retrospective comparative case series. Participants were identified using the procedural terminology codes. Groups were then matched using Coarsened Exact Matching (51 eyes in each group). The primary outcome measure was surgical success, defined as $5 \mathrm{mmHg}<\mathrm{IOP} \leq 21 \mathrm{mmHg}$, and IOP reduction $\geq 20 \%$ from baseline, and no reoperation for glaucoma. Secondary outcome measures were intraocular pressure, the number of glaucoma medications, and best corrected visual acuity (BCVA).

Results: The cumulative probability of success at one year was $61 \%$ in BT, and $50 \%$ in B. IOP decreased significantly from $23.5 \pm 2.4 \mathrm{mmHg}$ at baseline to $14.1 \pm 2.7 \mathrm{mmHg}$ at the final follow up in $\mathrm{BT}(P=0.001)$. The corresponding numbers for $B$ were $23.2 \pm 2.0$ and $13.9 \pm 1.6$, respectively $(P=0.001)$. There was no significant difference in IOP at the final follow-up $(P=0.56)$. The number of medications at baseline was $2.3 \pm 0.3$ in both groups. However, BT needed significantly fewer drops at all postoperative time intervals and used 1.1 $\pm 0.3(\mathrm{BT})$ and $2.0 \pm 0.4$ eye drops $(\mathrm{B})$ at the final follow-up visit $(\mathrm{P}=0.004)$. No dangerous hypotony or hypertension occurred in BT.

Conclusion: Similar rates of success and IOP reduction were observed in BT and B. BT needed significantly fewer glaucoma medications. Tube fenestration was not necessary in BT resulting in less postoperative hypotony and hypertension.

Keywords: Trabectome Surgery, ab interno trabeculectomy, glaucoma drainage devices, Baerveldt Glaucoma Implantation, tube ligation 


\section{Introduction}

Primary surgical interventions for refractory glaucoma with a low target IOP are often trabeculectomy and large glaucoma drainage devices (GDD). Both allow to bypass the impaired conventional outflow route and are therefore theoretically capable of achieving pressures below that of episcleral veins.

Trabeculectomy is associated with a relatively high rate of failure and potentially sight-threatening complications [1-4]. Recent studies indicate glaucoma drainage devices have the same or higher success rate than trabeculectomy in the management of end-stage glaucoma with a better safety profile $[1,2,5]$. As such, GDDs are increasingly used for refractory glaucomas or as primary procedures.

One of the most frequently implanted GDDs is the Baerveldt implant (Advanced Medical Optics, Santa Ana, California, USA). In contrast to the Ahmed glaucoma implant (New World Medical Inc, Rancho Cucamonga, California, USA), another commonly used device, the Baerveldt implant does not have a flow restrictor and requires a ligature suture that ties off the lumen completely [6] until a capsule has formed around the implant around four to six weeks [7] that limits flow sufficiently to prevent hypotony. Since GDDs are often implanted in severe cases of glaucoma with advanced optic neuropathy, a high postoperative IOP can be detrimental. Slit-shaped fenestrations are often created anterior to the ligature with spatulated needles to allow limited flow [8-10]. Unfortunately, this strategy can be poorly titrated and has a variable effect from no IOP reduction to frank hypotony [9].

To address this problem we combined Baerveldt device implantations with trabectome-mediated ab interno trabeculectomy surgery (NeoMedix, Tustin, CA, USA) to allow a complete tube ligature without fenestration. We hypothesized that this would provide appropriate IOP reduction until the ligature dissolves and the Baerveldt implant starts to function. To achieve a balanced comparison, we used Coarsened Exact Matching (CEM) a computation-intense, modern statistics method [11-14] as we have done before $[15,16]$. Although a randomized controlled trial remains the most effective tool to reduce bias and patient selection, modern statistical matching strategies can deliver a highly-balanced assessment of already existing, real-world patient data.

\section{Methods}

The protocol of this study was approved by the institutional review board of the University of Pittsburgh Human Subjects Research Committee (Approval number is: PRO13120466). An informed consent was not required for this retrospective comparative case series. Our research adhered to the tenets of the Declaration of Helsinki and regulations of the Health Insurance Portability and Accountability Act. Patients who underwent either Baerveldt with trabectome surgery (BT) or Baerveldt implant alone (B) between 2008 and 2015 were identified using Current Procedural Terminology codes. All procedures were 
performed by four glaucoma fellowship-trained surgeons. Patients older than 18 years old with medically uncontrolled IOP were included in this study. Exclusion criteria were neovascular glaucoma, uveitic glaucoma, and history of prior ocular surgery (except uncomplicated phacoemulsification).

Data were collected from patients' electronic medical record and included basic demographic information, type of glaucoma and preoperative diagnosis, preoperative IOP and number of glaucoma medications, baseline best corrected visual acuity (BCVA), type of operation, postoperative IOP, number of medications, and BCVA.

The primary outcome measure was success defined as $5 \mathrm{mmHg}<\mathrm{IOP} \leq 21 \mathrm{mmHg}$ or $\geq 20 \%$ reduction of IOP from baseline at two consecutive visits, no need for further glaucoma surgery, and no loss of light perception. The secondary outcome measures were IOP, BCVA, and the number of medications.

In all cases, the IOP was measured with Goldmann applanation tonometer (GAT; Haag-Streit, Konig, Switzerland) at 1 day, 1 week, $4 \pm 1$ week, 2-4 months, 5-7 months, 8-10 months, and 11-13 months. If more than one visit was found at these intervals, the visit closest to month 6, 9, or 12 was chosen.

\section{Surgical technique}

In BT, trabectome surgery was performed first. The patient's head was tilted $30^{\circ}$ away from the surgeon and the microscope was tiled in the opposite direction. A temporal $1.6 \mathrm{~mm}$ clear corneal incision was created, and the handpiece was advanced into the anterior chamber. The tip of the trabectome was engaged with trabecular meshwork (TM) at the nasal angle and advanced parallel to Schlemm's canal for $90^{\circ}$ counterclockwise followed by a $90^{\circ}$ clockwise move to opposite direction for a total of $180^{\circ} \mathrm{TM}$ ablation. The handpiece was withdrawn from the anterior chamber, and the incision was hydrated to seal.

Baerveldt implantation: a fornix-based conjunctival flap was created, and tenon's dissection was advanced toward the equator until enough space was created for the implant. The lateral wings of the $350 \mathrm{~mm} * \mathrm{~mm}$ Baerveldt implant were advanced under the superior and lateral rectus muscles. The plate was sutured to the sclera $10 \mathrm{~mm}$ posterior to the limbus. The tube was cut short with the bevel up to have approximately 2-3 mm intracameral length. The tube was completely ligated near the plate junction with a 7-0 polyglactin 910 suture (coated VICRYL, Ethicon, Somerville, NJ, United States) and tested with BSS to confirm water tightness. The tube was then inserted into the anterior chamber through a tunnel created with 23-gauge needle and secured to the sclera with a 7-0 polyglactin loop stitch. In B, but not in BT, the tube portion anterior to the ligature was fenestrated with a pass of the spatulated 7-0 needle. A patch graft was used to cover the tube. The conjunctiva and Tenon's layer were pulled back over the shunt and sutured to the limbus. 
At the conclusion of the surgery, an antibiotic (moxifloxacin) and steroid (1\% prednisolone acetate) drop were applied. The antibiotic was used four times per day for one week while the steroid eye drops were used four times per day for one month and then tapered by one drop application per week.

\section{Statistical analysis}

Demographics were compared by the Mann-Whitney $U$ test and chi-squared test for continuous and categorical variables, respectively. To avoid eliminating data with missing values, Multiple Imputation in $\mathrm{R}$ was used. Missing values of the incomplete dataset were imputed $m>1$ times, thus creating $m$ completed datasets. Second, each of the $m$ completed datasets were independently analyzed. Finally, the results from each of the $m$ analysis were pooled into a final result. Missing data like age, gender, and race were imputed by generating five similar but non-identical datasets. Groups were then matched by utilizing Coarsened Exact Matching in R [17], based on age, race, type of glaucoma, baseline IOP, and number of preoperative glaucoma medications.

Univariate linear regression was used to examine IOP reduction after surgery. Variables statistically significant were included in the final multivariate regression model. A p-value of less than 0.05 was considered statistically significant. Continuous variables were expressed as mean $\pm S D$. All analyses were performed using $\mathrm{R}$.

To compute the survival of subjects in the groups, Kaplan-Meier survival plots were determined and compared using the log-rank test. Statistical significance was set at $p<0.05$. Success was defined as the IOP $<21 \mathrm{mmHg}$ and a $>20 \%$ reduction from baseline with no need to further surgery.

\section{Results}

175 eyes were included consisting of 51 BT and 51 B. Using case matching, there was no significant difference in ethnicity, IOP, the number of IOP-lowering medications, glaucoma type, the degree of VF loss and GI between AIT and AGI ( $p>0.05)$. Table 1 shows the baseline characteristics of each group.

The mean age of the study participants was $70.7 \pm 11.1$ years in $B T$ and $67.2 \pm 15.7$ years in $B(P=0.116)$. Thirty patients (59\%) underwent phacoemulsification at the time of glaucoma surgery in each group $(P=1.00)$. Primary open-angle glaucoma was the most common diagnosis in both groups (65.0\% and $56.5 \%$ in $B T$ and $B$, respectively, $P=0.516$ ) There were no significant differences between the study groups in terms of gender, preoperative intraocular pressure, baseline number of glaucoma medications, ethnicity, and type of glaucoma (Table 1). 
Kaplan-Meier survival curves (Figure 1) indicated a mean duration of survival of $261.9 \pm 21.9$ days in BT group and 220.28 \pm 17.5 in B group with no statistically significant difference between two groups (log rank=2.53 $p=0.11$ ). The cumulative probability of qualified success at 3 months, 6 months, and 12 months was $74 \%, 64 \%$, and $61 \%$ respectively in the $\mathrm{BT}$, and $66 \%, 52 \%$, and $50 \%$ in the $\mathrm{B}$.

In subgroup analysis, although BT combined with phacoemulsification had longer survival than B +phacoemulsification, the difference was not statistically significant. The mean survival duration was $285.5 \pm 25.8$ in BT with phacoemulsification versus $225.8 \pm 25.9$ in B with phacoemulsification, log Rank= $2.17, \mathrm{P}=0.14)$. Corresponding values for glaucoma surgery alone were $221 \pm 37.2$ and $215.5 \pm 23.6$ in $\mathrm{BT}$ and $B$ group, respectively (log-Rank $=0.24, p=0.624$, Figure 1$)$. BT combined with phacoemulsification had longer survival than BT, but the difference was not statistically significant (285.5 \pm 25.8 versus $221 \pm 37.2$, Log-Rank= 1.18 P=0.18). Corresponding values for $B$ were $225.8 \pm 25.9$ and $215.5 \pm 23.6$ in $B$ with phacoemulsification and B alone, respectively (log-Rank $=0.45, \mathrm{P}=0.50)$.

IOP was decreased significantly from $23.5 \pm 2.4 \mathrm{mmHg}$ at baseline to $14.1 \pm 2.7 \mathrm{mmHg}$ at final follow up in BT $(P=0.001$, Figure 2). The corresponding numbers for $B$ were $23.2 \pm 2.0$ and $13.9 \pm 1.6$, respectively $(P=$ 0.001). IOP varied more in B than in BT during the early postoperative phase with $6.3 \%$ of hypotony in BT versus $12.8 \%$ hypotony in B. During the 1-year follow-up, 7 (13.7\%) patients in BT and $18(35.2 \%)$ patients in $B$ group experienced hypotony $(P=0.04)$. Most of the hypotony episodes were within the first month before suture opening.

There was no significant difference in IOP at final follow-up $(P=0.98)$. Eyes in BT experienced a $9 \pm 9.1$ $\mathrm{mmHg}$ reduction in IOP within one week after the surgery compared to a $6 \pm 12.3 \mathrm{mmHg}$ reduction in $B(P=$ 0.09). IOP was comparable on day one between BT with phacoemulsification and BT $(20.3 \pm 11.1 \mathrm{mmHg}$ versus $18.6 \pm 12.7 \mathrm{mmHg}, \mathrm{P}=0.56)$. B with phacoemulsification had a significantly higher IOP on day 1 compared to $B(23.2 \pm 14.3$ versus $17.9 \pm 11.4, P=0.041)$.

The baseline number of glaucoma medications was $2.3 \pm 0.3$ in both groups (Figure 3). However, BT needed statistically significantly fewer drops in all postoperative visits. At the final follow-up visit, the number of glaucoma medications was $1.1 \pm 0.3$ drops in $B T$ and $2.0 \pm 0.4$ in $B(p=0.003$, Figure 3$)$.

The mean BCVA at the baseline was $0.64 \pm 0.85 \log M A R$ in $B T$ and $0.55 \pm 0.75 \log M A R$ in $B(P=0.663)$. Corresponding numbers for final follow-up visit was $0.72 \pm 1.07 \log M A R$ and $0.63 \pm 0.97 \log M A R$, respectively $(P=0.668)$.

\section{Discussion}


BT and B were both effective in reducing IOP. The IOP reduction of 31\% at the 1-year follow-up was similar to previous reports [18-20]. There was a trend toward greater IOP reduction following BT compared to $B$, but the difference did not reach statistical significance. However, BT required significantly fewer medications postoperatively. The number of glaucoma medications at 1 month postoperative visit was nearly three times as high in B as surgeons struggled to control the pressure in this phase of the bleb maturation.

Early complications of glaucoma drainage devices include hyphema, shallow or flat anterior chamber, tube-corneal touch, corneal edema, and suprachoroidal effusion [6, 21]. These complications are caused by postoperative hypotony, more common in non-valved devices when the flow is not restricted and when fenestration of the tube yields excessive flow [22]. Complete ligation of the tube can prevent postoperative hypotony [23-26], but high postoperative IOPs can be dangerous to eyes with advanced glaucoma damage. Therefore, tube ligation is mostly carried out in conjunction with intraoperative longitudinally oriented $2 \mathrm{~mm}$ fenestrations proximal to the ligation [22]. Despite this, a postoperative hypertensive spike may develop secondary to fibrotic growth around the fenestration, malfunction of the slit valves, or insufficient fenestrations $[8,27]$. Considering the limitations of fenestration, we proposed to perform trabectome surgery in the same session to prevent postoperative IOP spikes. Trabectome enhances outflow by plasma-mediated ablation of the trabecular meshwork and has a long track record of efficacy and safety in several types of glaucomas [28-32].

Although trabectome is in the family of minimally invasive glaucoma surgeries and primarily proposed for mild open-angle glaucoma cases [32], recent studies suggest it is also effective in more severe glaucomas [33] but it requires a patent conventional drainage system downstream of the trabecular meshwork. Although the success rate of trabectome after failed trabeculectomy and tube shunt procedure supports its role in the management of severe glaucoma [34, 35], many eyes at that stage cannot afford a surgical failure. The effect of trabectome is immediate and controls IOP until absorbable ligation sutures dissolve, and $B$ begins to function. In contrast to fenestration, the IOP lowering effect of trabectome also persists after the ligature suture is absorbed and has the additive effect of reducing the need for glaucoma medications. This is no small feat as nearly half of all glaucoma patients experience local and systemic side effects of glaucoma medications [36]. Adverse effects from medication are an important reason for nonadherence [37] and can also jeopardize the success of glaucoma surgery [38,39]. Conversely, reducing eye drops can considerably improve the quality of life [40].

Since cataract and glaucoma frequently coexist, many individuals in both groups underwent cataract surgery concurrently. IOP in BT with phacoemulsification was not significantly different from BT alone, reflecting our prior results that phacoemulsification does not add to the IOP lowering effect when 
combined with trabectome surgery [15]. Although phacoemulsification has been advocated as providing a trabeculoplasty-like, additional IOP reduction [41], glaucomatous trabecular meshwork is often unpredictable and can result in IOP spikes $[42,43]$. We observed this on day 1 in patients in B with phacoemulsification. The results of our study and previous reports on this subject show that this potentially dangerous IOP spike could be prevented by the same session trabectome surgery [44]. Conversely, no severe hypotony was seen in BT that did not require tube fenestration.

Limitations of this study are inherent to the retrospective nature compared to randomized controlled trials. However, the CEM strategy used here reduces imbalances without discarding valid data. Although observational data is easy to collect compared to randomized experiments, how the treatments were assigned and other aspects of data generation are often ambiguous and difficult to control. CEM is a newer form of automatic, nonparametric matching to control the confounding influence of pretreatment control variables by achieving an acceptable balance between treated and control groups [17].

Additionally, this study was conducted at a single tertiary academic center, so the results cannot easily be generalized to other practice facilities.

In summary, we found that Baerveldt implants with same-session trabectome surgery had a significantly decreased number of glaucoma medications and avoided both severe hypertension and hypotension, thereby negating the need for tube fenestration.

\section{Funding}

We acknowledge support from The Initiative to Cure Glaucoma, The Eye and Ear Foundation of Pittsburgh; NIH CORE Grant P30 EY08098 to the Department of Ophthalmology; an unrestricted grant from Research to Prevent Blindness, New York, NY 


\section{References}

1. Gedde SJ, Schiffman JC, Feuer WJ, et al (2012) Treatment outcomes in the Tube Versus Trabeculectomy (TVT) study after five years of follow-up. Am J Ophthalmol 153:789-803.e2

2. Gedde SJ, Herndon LW, Brandt JD, et al (2012) Postoperative complications in the Tube Versus Trabeculectomy (TVT) study during five years of follow-up. Am J Ophthalmol 153:804-814.e1

3. Esfandiari H, Pakravan M, Loewen NA, Yaseri M (2017) Predictive value of early postoperative IOP and bleb morphology in Mitomycin-C augmented trabeculectomy. F1000Res 6: . doi: 10.12688/f1000research.12904.1

4. Pakravan M, Esfandiari H, Yazdani S, et al (2017) Mitomycin C-augmented trabeculectomy: subtenon injection versus soaked sponges: a randomised clinical trial. Br J Ophthalmol 101:1275-1280

5. Tseng VL, Coleman AL, Chang MY, Caprioli J (2017) Aqueous shunts for glaucoma. Cochrane Database Syst Rev 7:CD004918

6. Patel S, Pasquale LR (2010) Glaucoma drainage devices: a review of the past, present, and future. Semin Ophthalmol 25:265-270

7. Minckler DS, Francis BA, Hodapp EA, et al (2008) Aqueous shunts in glaucoma: a report by the American Academy of Ophthalmology. Ophthalmology 115:1089-1098

8. Kansal S, Moster MR, Kim D, et al (2002) Effectiveness of nonocclusive ligature and fenestration used in Baerveldt aqueous shunts for early postoperative intraocular pressure control. J Glaucoma 11:6570

9. Ortiz Arismendi GE, Peña Valderrama CDP, Albis-Donado O (2013) Results of a New Technique for Implantation of Nonrestrictive Glaucoma Devices. J Curr Glaucoma Pract 7:130-135

10. Trible JR, Brown DB (1998) Occlusive ligature and standardized fenestration of a Baerveldt tube with and without antimetabolites for early postoperative intraocular pressure control. Ophthalmology 105:2243-2250

11. lacus SM, King G, Porro G (2009) CEM: Software for coarsened exact matching. J Stat Softw 30:1-27

12. lacus SM, King G, Porro G (2012) Causal Inference without Balance Checking: Coarsened Exact Matching. Polit Anal 20:1-24

13. Rubin DB, Thomas N (2000) Combining Propensity Score Matching with Additional Adjustments for Prognostic Covariates. J Am Stat Assoc 95:573-585

14. Seeger JD, Williams PL, Walker AM (2005) An application of propensity score matching using claims data. Pharmacoepidemiol Drug Saf 14:465-476

15. Parikh HA, Bussel II, Schuman JS, et al (2016) Coarsened Exact Matching of Phaco-Trabectome to Trabectome in Phakic Patients: Lack of Additional Pressure Reduction from Phacoemulsification. PLoS One 11:e0149384 
16. Neiweem AE, Bussel II, Schuman JS, et al (2016) Glaucoma Surgery Calculator: Limited Additive Effect of Phacoemulsification on Intraocular Pressure in Ab Interno Trabeculectomy. PLoS One 11:e0153585

17. lacus SM, King G, Porro G (2012) Causal Inference without Balance Checking: Coarsened Exact Matching. Polit Anal 20:1-24

18. Kostanyan T, Shazly T, Kaplowitz KB, et al (2017) Longer-term Baerveldt to Trabectome glaucoma surgery comparison using propensity score matching. Graefes Arch Clin Exp Ophthalmol. doi: 10.1007/s00417-017-3804-9

19. Christakis PG, Zhang D, Budenz DL, et al (2017) Five-Year Pooled Data Analysis of the Ahmed Baerveldt Comparison Study and the Ahmed Versus Baerveldt Study. Am J Ophthalmol 176:118-126

20. Christakis PG, Kalenak JW, Tsai JC, et al (2016) The Ahmed Versus Baerveldt Study: Five-Year Treatment Outcomes. Ophthalmology 123:2093-2102

21. Budenz DL, Barton K, Feuer WJ, et al (2011) Treatment outcomes in the Ahmed Baerveldt Comparison Study after 1 year of follow-up. Ophthalmology 118:443-452

22. Aref AA, Gedde SJ, Budenz DL (2012) Glaucoma drainage implant surgery. Dev Ophthalmol 50:37-47

23. Molteno AC, Van Biljon G, Ancker E (1979) Two-stage insertion of glaucoma drainage implants. Trans Ophthalmol Soc N Z 31:17-26

24. Molteno AC, Polkinghorne PJ, Bowbyes JA (1986) The vicryl tie technique for inserting a draining implant in the treatment of secondary glaucoma. Aust N Z J Ophthalmol 14:343-354

25. Egbert PR, Lieberman MF (1989) Internal suture occlusion of the Molteno glaucoma implant for the prevention of postoperative hypotony. Ophthalmic Surg 20:53-56

26. Sherwood MB, Smith MF (1993) Prevention of early hypotony associated with Molteno implants by a new occluding stent technique. Ophthalmology 100:85-90

27. Campbell RJ, Buys YM, Mcllraith IP, Trope GE (2008) Internal glaucoma drainage device tube fenestration for uncontrolled postoperative intraocular pressure. J Glaucoma 17:494-496

28. Kaplowitz K, Bussel II, Honkanen R, et al (2016) Review and meta-analysis of ab-interno trabeculectomy outcomes. Br J Ophthalmol 100:594-600

29. Ngai P, Kim G, Chak G, et al (2016) Outcome of primary trabeculotomy ab interno (Trabectome) surgery in patients with steroid-induced glaucoma. Medicine 95:e5383

30. Ting JLM, Damji KF, Stiles MC, Trabectome Study Group (2012) Ab interno trabeculectomy: outcomes in exfoliation versus primary open-angle glaucoma. J Cataract Refract Surg 38:315-323

31. Bussel II, Kaplowitz K, Schuman JS, et al (2015) Outcomes of ab interno trabeculectomy with the trabectome by degree of angle opening. Br J Ophthalmol 99:914-919

32. Minckler DS, Baerveldt G, Alfaro MR, Francis BA (2005) Clinical results with the Trabectome for treatment of open-angle glaucoma. Ophthalmology 112:962-967 
33. Loewen RT, Roy P, Parikh HA, et al (2016) Impact of a Glaucoma Severity Index on Results of Trabectome Surgery: Larger Pressure Reduction in More Severe Glaucoma. PLoS One 11:e0151926

34. Bussel II, Kaplowitz K, Schuman JS, et al (2015) Outcomes of ab interno trabeculectomy with the trabectome after failed trabeculectomy. Br J Ophthalmol 99:258-262

35. Mosaed S, Chak G, Haider A, et al (2015) Results of Trabectome Surgery Following Failed Glaucoma Tube Shunt Implantation: Cohort Study. Medicine 94:e1045

36. Pisella PJ, Pouliquen P, Baudouin C (2002) Prevalence of ocular symptoms and signs with preserved and preservative free glaucoma medication. $\mathrm{Br} J$ Ophthalmol 86:418-423

37. Tsai JC, McClure CA, Ramos SE, et al (2003) Compliance barriers in glaucoma: a systematic classification. J Glaucoma 12:393-398

38. Lavin MJ, Wormald RP, Migdal CS, Hitchings RA (1990) The influence of prior therapy on the success of trabeculectomy. Arch Ophthalmol 108:1543-1548

39. Broadway D, Grierson I, Hitchings R (1993) Adverse effects of topical antiglaucomatous medications on the conjunctiva. Br J Ophthalmol 77:590-596

40. Loon SC, Jin J, Jin Goh M (2015) The relationship between quality of life and adherence to medication in glaucoma patients in Singapore. J Glaucoma 24:e36-42

41. Poley BJ, Lindstrom RL, Samuelson - Journal of Cataract \& ... TW, 2009 (2009) Intraocular pressure reduction after phacoemulsification with intraocular lens implantation in glaucomatous and nonglaucomatous eyes: evaluation of a .... Elsevier Oceanogr Ser

42. Slabaugh MA, Bojikian KD, Moore DB, Chen PP (2014) Risk factors for acute postoperative intraocular pressure elevation after phacoemulsification in glaucoma patients. J Cataract Refract Surg 40:538544

43. Weiner $Y$, Severson $M L$, Weiner $A$ (2015) Intraocular pressure 3 to 4 hours and 20 hours after cataract surgery with and without ab interno trabeculectomy. J Cataract Refract Surg 41:2081-2091

44. Neiweem AE, Bussel II, Schuman JS, et al (2016) Glaucoma Surgery Calculator: Limited Additive Effect of Phacoemulsification on Intraocular Pressure in Ab Interno Trabeculectomy. PLoS One 11:e0153585

\section{Tables}


Table 1. Baseline clinical characteristics of patients in BT and B group

\begin{tabular}{|c|c|c|c|c|}
\hline & & \multicolumn{2}{|l|}{ Group } & \multirow[b]{2}{*}{$\mathbf{P}$} \\
\hline & & BT & B & \\
\hline Age & Mean $\pm S D$ & $70.7 \pm 11.1$ & $67.2 \pm 15.7$ & $0.116^{\dagger}$ \\
\hline BCVA & Mean $\pm S D$ & $0.64 \pm 0.85$ & $0.55 \pm 0.75$ & $0.663 \ddagger$ \\
\hline IOP & Mean $\pm S D$ & $23.4 \pm 2.4$ & $22.8 \pm 2.1$ & $0.871^{\dagger}$ \\
\hline Medications & Mean $\pm S D$ & $2.3 \pm 0.3$ & $2.3 \pm 0.3$ & $1.00 \ddagger$ \\
\hline \multirow[t]{2}{*}{ Gender } & Female & $32(63 \%)$ & 27 (53\%) & \\
\hline & Male & $24(47 \%)$ & 55 (47.8\%) & $0.32^{*}$ \\
\hline \multirow[t]{2}{*}{ Phaco } & Yes & $30(59 \%)$ & 30 (59\%) & $1.00^{*}$ \\
\hline & No & $21(41 \%)$ & $21(41 \%)$ & \\
\hline \multirow[t]{3}{*}{ Ethnicity } & White & 37 (61.7\%) & 70 (60.9\%) & $0.99 * *$ \\
\hline & African American & 21 (35.0\%) & 42 (36.5\%) & \\
\hline & Other & $2(3.3 \%)$ & $3(2.6 \%)$ & \\
\hline \multirow[t]{5}{*}{ Glaucoma } & POAG & 39 (65.0\%) & 65 (56.5\%) & \\
\hline & XFG & $3(5.0 \%)$ & $8(7.0 \%)$ & \\
\hline & PACG & 7 (11.7\%) & $10(8.7 \%)$ & $0.516^{* *}$ \\
\hline & PG & $3(5.0 \%)$ & $5(4.3 \%)$ & \\
\hline & Other & 8 (13.3\%) & 27 (23.5\%) & \\
\hline
\end{tabular}

BCVA: best corrected visual acuity; IOP: intraocular pressure; POAG: primary open angle glaucoma; XFG: exfoliation glaucoma; PACG: primary angle closure glaucoma; PG: pigmentary glaucoma. ch ${ }^{\dagger}$ Based on $\mathrm{t}-$ test. ¥ Based on Mann-Whitney test. * Based on Chi-Square test.** Based on Fisher exact test. 


\section{Figures}

Figure 1

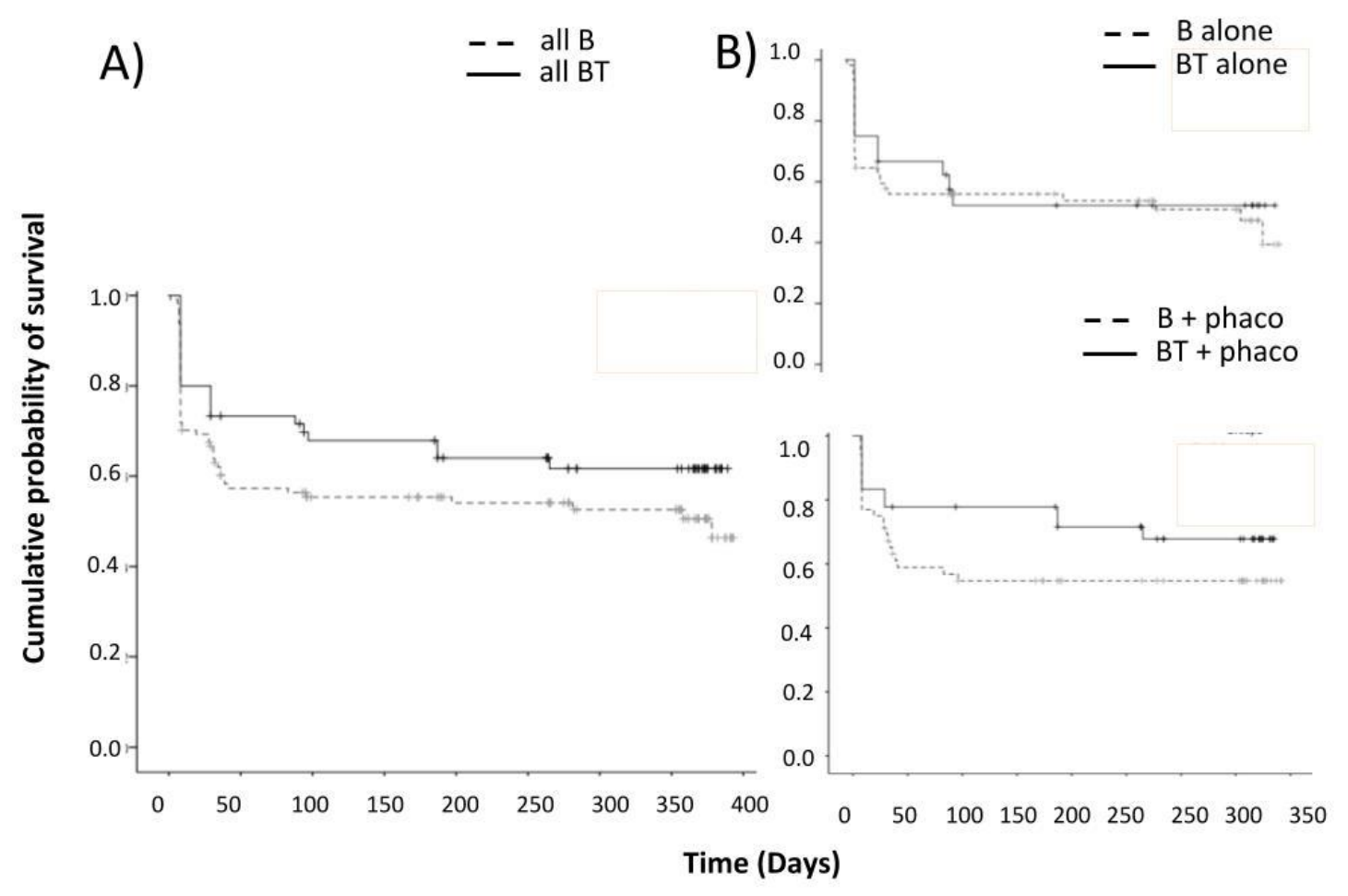

Figure 1. A) Kaplan-Meier survival plots for BT and B with success defined as a final IOP of $\leq 21 \mathrm{mmHg}$ and a $20 \%$ reduction from baseline. Success rates of (BT) and B was similar in both groups. B) survival plots of BT and B for subgroup analysis separated by B) glaucoma surgery alone and C) same session phacoemulsification (lower right). 


\section{Figure 2}
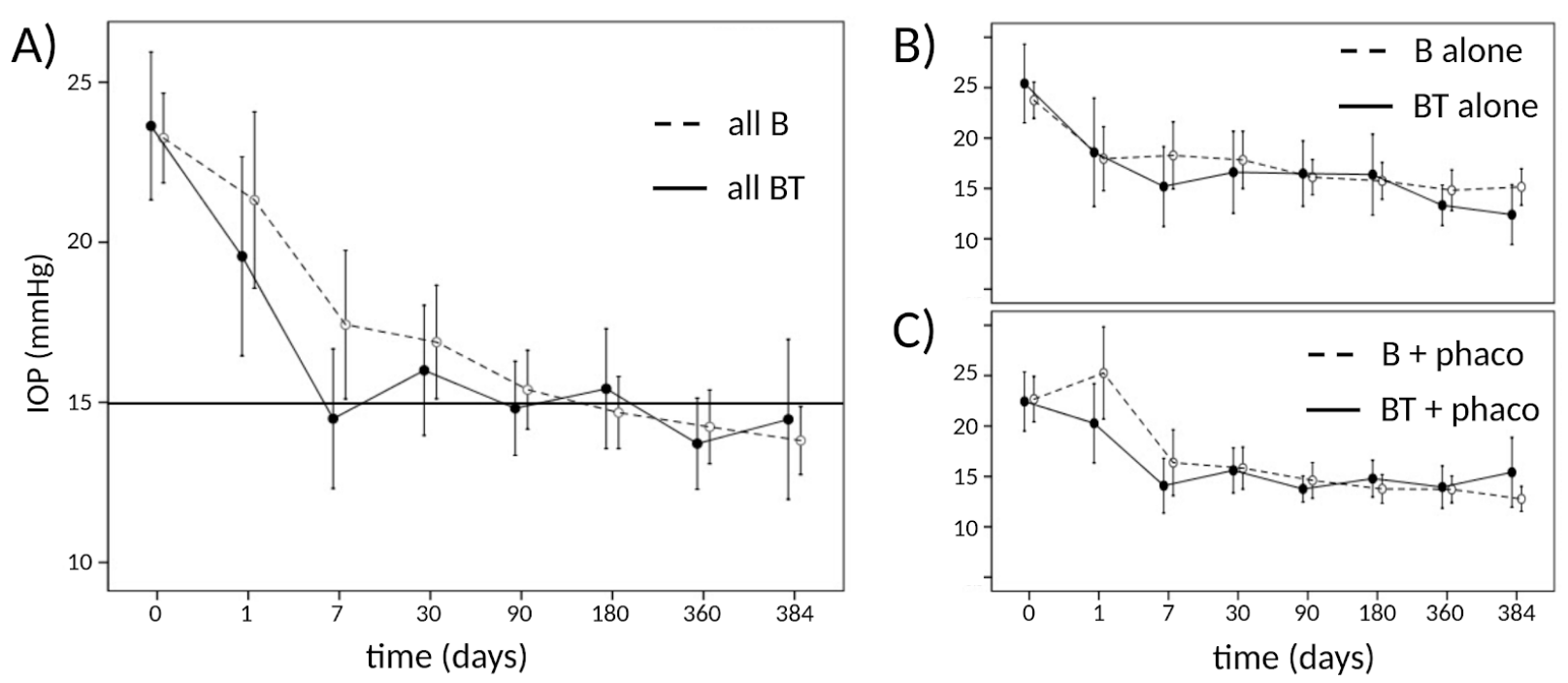

Figure 2. IOP in $B$ and $B T$. A) IOP in BT was similar to $B$ and trended towards a lower average although tubes in BT were not fenestrated and trended towards a lower average IOP. B) B and BT as standalone procedures. C) B + phaco had a higher IOP on day one compared to subsequent IOPs. No such peak was seen in BT. Mean $\pm 95 \%$ confidence interval. 


\section{Figure 3}
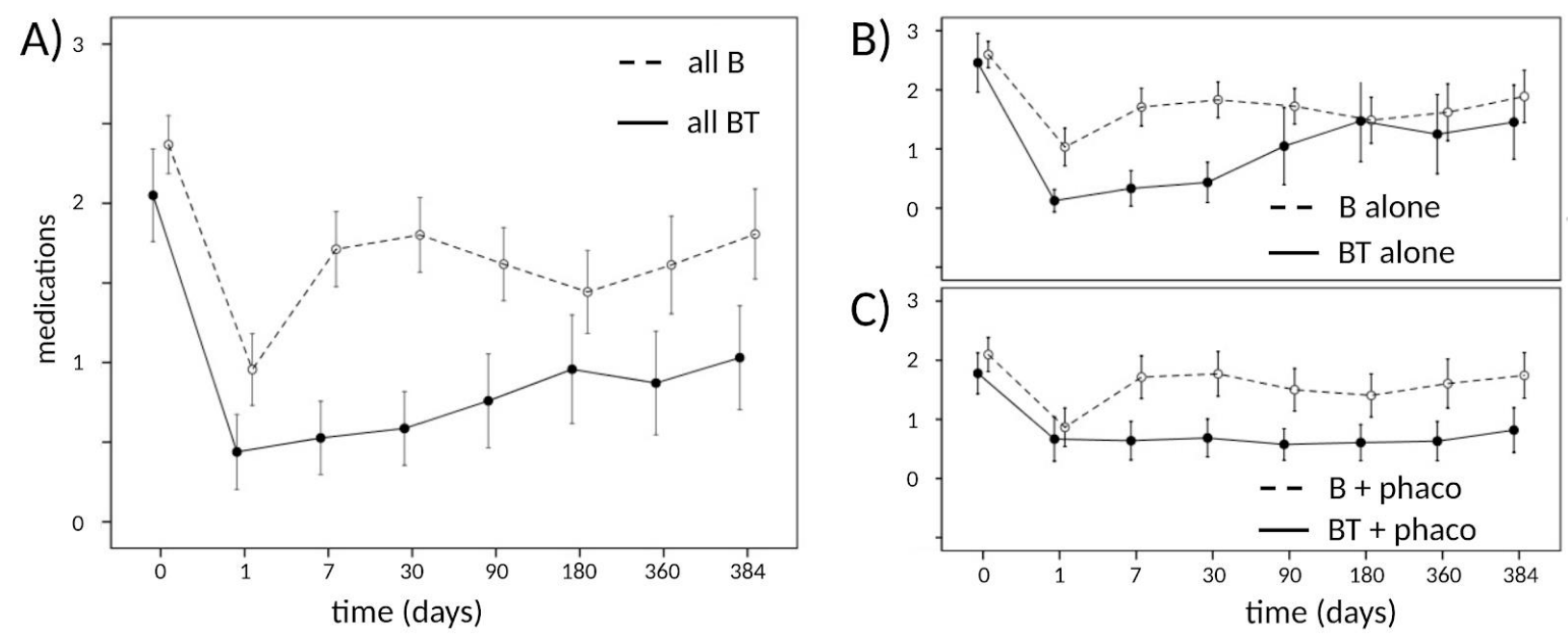

Figure 3. Preoperative and postoperative mean eyedrops for BT and B. Mean pre- and postoperative glaucoma medications for subgroup analysis separated by glaucoma surgery only (B) and same session phacoemulsification (C). Represented as mean $\pm 95 \%$ confidence Intervals. 